\title{
Neuronal uptake transporters contribute to oxaliplatin neurotoxicity in mice
}

Kevin M. Huang, ${ }^{1}$ Alix F. Leblanc, ${ }^{1}$ Muhammad Erfan Uddin, ${ }^{1}$ Ji Young Kim, ${ }^{1}$ Mingqing Chen, ${ }^{1}$ Eric D. Eisenmann, ${ }^{1}$ Alice A. Gibson, ${ }^{1}$ Yang Li, ${ }^{1}$ Kristen W. Hong, ${ }^{1}$ Duncan DiGiacomo, ${ }^{1}$ Sherry H. Xia, ${ }^{1}$ Paola Alberti, ${ }^{2,3}$ Alessia Chiorazzi, ${ }^{2,3}$ Stephen N. Housley, ${ }^{4}$ Timothy C. Cope, ${ }^{4}$ Jason A. Sprowl, ${ }^{5}$ Jing Wang, ${ }^{6}$ Charles L. Loprinzi, ${ }^{7}$ Anne Noonan, ${ }^{8}$ Maryam B. Lustberg, ${ }^{8}$ Guido Cavaletti, ${ }^{2,3}$ Navjot Pabla, ${ }^{1}$ Shuiying Hu, ${ }^{1}$ and Alex Sparreboom ${ }^{1}$

'Division of Pharmaceutics and Pharmacology, College of Pharmacy and Comprehensive Cancer Center, The Ohio State University, Columbus, Ohio, USA. ${ }^{2}$ School of Medicine and Surgery, University of Milano-Bicocca, Monza, Italy. ${ }^{3}$ NeuroMI, Milan Center for Neuroscience, Milan, Italy. ${ }^{4}$ School of Biological Sciences and Coulter Department of Biomedical Engineering, Ceorgia Institute of Technology, Atlanta, Georgia, USA. ${ }^{5}$ Department of Pharmaceutical Sciences, School of Pharmacy and Pharmaceutical Sciences, University at Buffalo, Buffalo, New York, USA. ${ }^{6}$ Department of Cancer Biology and Genetics, College of Medicine and Comprehensive Cancer Center, The Ohio State University, Columbus, Ohio, USA. 'Department of Oncology, Mayo Clinic Comprehensive Cancer Center, Rochester, Minnesota, USA. ${ }^{8}$ Division of Medical Oncology, Department of Internal Medicine, College of Medicine and Comprehensive Cancer Center, The Ohio State University, Columbus, Ohio, USA.

\begin{abstract}
Peripheral neurotoxicity is a debilitating condition that afflicts up to $90 \%$ of patients with colorectal cancer receiving oxaliplatin-containing therapy. Although emerging evidence has highlighted the importance of various solute carriers to the toxicity of anticancer drugs, the contribution of these proteins to oxaliplatin-induced peripheral neurotoxicity remains controversial. Among candidate transporters investigated in genetically engineered mouse models, we provide evidence for a critical role of the organic cation transporter 2 (OCT2) in satellite glial cells in oxaliplatin-induced neurotoxicity, and demonstrate that targeting OCT2 using genetic and pharmacological approaches ameliorates acute and chronic forms of neurotoxicity. The relevance of this transport system was verified in transporter-deficient rats as a secondary model organism, and translational significance of preventive strategies was demonstrated in preclinical models of colorectal cancer. These studies suggest that pharmacological targeting of OCT2 could be exploited to afford neuroprotection in cancer patients requiring treatment with oxaliplatin.
\end{abstract}

\section{Introduction}

Clinical use of the chemotherapeutic agent oxaliplatin is associated with a characteristic pattern of peripheral neurotoxicity that affects more than $90 \%$ of patients (1). The onset of neurotoxicity may occur immediately after infusion and is characterized by cold-exacerbated paresthesias, muscle spasms, and fasciculations. Although these acute symptoms resolve over time, at higher cumulative doses, oxaliplatin induces dose-limiting sensory neurotoxicity that leads to functional impairment, which can last for years following the discontinuation of treatment and, in more severe cases, result in permanent impairment (2).

The mechanisms underlying oxaliplatin-induced peripheral neurotoxicity (OIPN) remain incompletely understood. Within the nervous system, oxaliplatin preferentially accumulates in peripheral sensory neurons present in the dorsal root ganglion (DRG) (3), and oxaliplatin levels in DRGs remain high for prolonged time periods, even after discontinuation of treatment. Because the severity of OIPN correlates with drug levels

Authorship note: $\mathrm{KMH}$ and $\mathrm{AFL}$ contributed equally to the manuscript. Conflict of interest: MBL has provided consultation regarding chemotherapy-induced peripheral neuropathy to PledPharma and Disarm Therapeutics. CLL has provided consultation regarding chemotherapy-induced peripheral neuropathy to PledPharma, Disarm Therapeutics, Asahi Kasei, and Metys Pharmaceuticals. Copyright: (5) 2020, American Society for Clinical Investigation. Submitted: January 30, 2020; Accepted: May 15, 2020; Published: July 27, 2020 Reference information: J Clin Invest. 2020;130(9):4601-4606. https://doi.org/10.1172/JCl136796. in peripheral nerves (4), these studies suggest that oxaliplatin accumulation in DRGs is a key trigger for the development of OIPN, and that pharmacological targeting of the transport mechanism regulating the initial accumulation of oxaliplatin could be a promising, previously unexplored, neuroprotective strategy. Studies have demonstrated that the cellular uptake of platinum-based chemotherapeutics such as cisplatin occurs via a facilitated transport mechanism (5). Consequently, distribution patterns and pathological changes following oxaliplatin administration are restricted to cells in organs capable of transporting oxaliplatin from the blood into cells $(6,7)$. Previous reports have demonstrated that the organic cation transporter 2 (OCT2, encoded by SLC22A2) mediates the intracellular accumulation of both cisplatin and oxaliplatin in mice, rats, and humans (7-10), and several other cationic-type transporters (zwitterion transporters [OCTNs] and multidrug and toxin extrusion proteins [MATEs]) and organic anion-transporting polypeptides (OATPs), may contribute to this process $(6,11-13)$.

The aim of the present study was to unambiguously identify the DRG transporter mediating oxaliplatin accumulation with the use of engineered knockout mice and rats, and establish the feasibility of a strategic intervention concept with translational potential using transport inhibitors. This approach is derived from the supposition that targeting of an initial uptake mechanism offers conceptual advances over interventions involving intracellular neuronal signaling cascades that may also be relevant to oxaliplatin-mediated antitumor efficacy. 


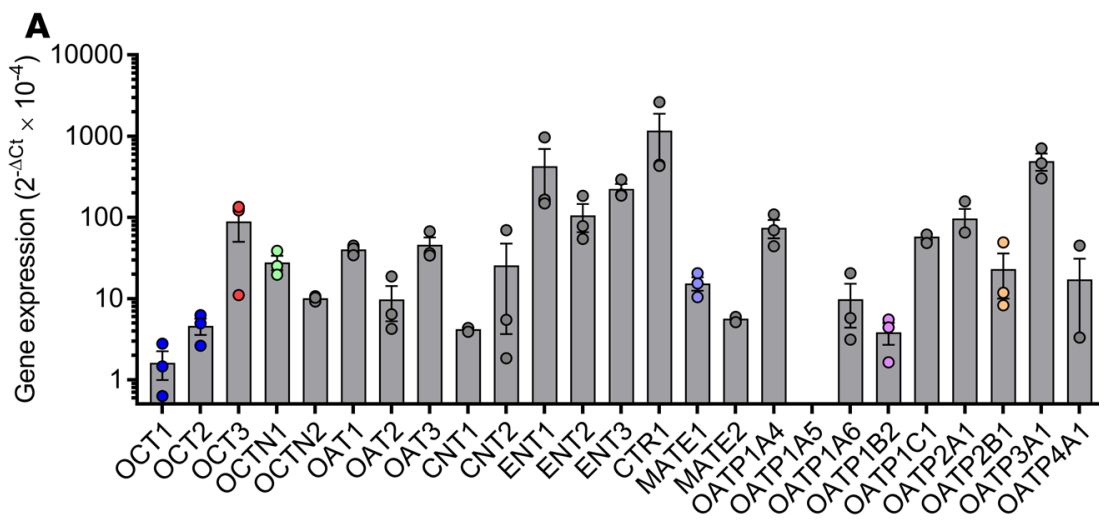

B
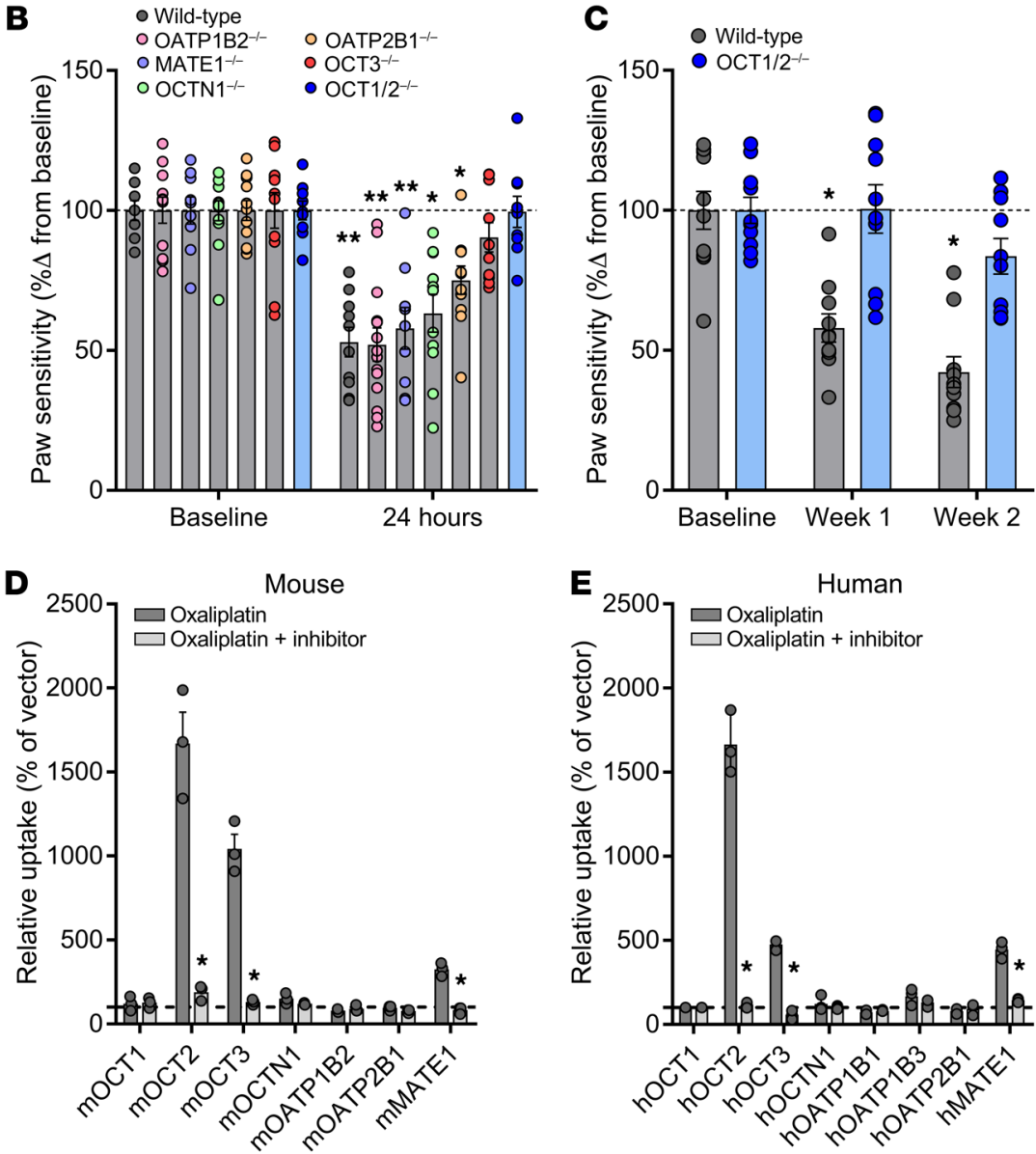

Figure 1. OCT2 is a critical determinant of OIPN. (A)

Transcriptomic expression of transporters in DRGs isolated from untreated WT FVB mice. (B) Acute OIPN in WT (pooled C57BL/6, FVB, or DBA strains) or transporter-deficient mice 24 hours following a single injection of $10 \mathrm{mg} / \mathrm{kg}$ oxaliplatin. (C) Chronic OIPN in WT or OCT $1 / 2^{-1-}$ mice 1 or 2 weeks following multiple injections of $4 \mathrm{mg} / \mathrm{kg}$ oxaliplatin, twice a week (cumulative dose $32 \mathrm{mg} / \mathrm{kg}$ ). Paw sensitivity is expressed as percentage change relative to baseline values. (D and E) Intracellular accumulation and inhibition (for specific inhibitors, please refer to Supplemental Methods) of oxaliplatin uptake into HEK293 cells overexpressing (D) mouse (m) or (E) human (h) transporters. Relative uptake is expressed as percentage change compared with empty vector controls. All animal studies contained $n=4-8$ per group, repeated on 2 independent occasions. All in vitro studies contained $n=3$ per group, repeated on 3 independent occasions. Statistical analysis was performed using an unpaired, 2-sided Student's $t$ test with Welch's correction: ${ }^{*} P<$ $0.05,{ }^{*} P<0.01$ compared with baseline values.

on observed expression signatures and putative relevance, OCT1 and OCT2 (hereafter OCT1/2), OCT3, OCTN1, OATP1B2, OATP2B1, and MATE1 were selected for further consideration as in vivo transporters of oxaliplatin. A comparative von Frey hair test was used in WT and transporter-deficient mice to assess neuropathy 24 hours following a single dose of oxaliplatin. OCT1/2 deficiency conferred significant protection against OIPN in both acute and chronic models (Figure 1, B and C). The observed phenotypic changes occurred without concurrent alteration of oxaliplatin pharmacokinetics caused by OCT1/2 deficiency, as described previously (9). In addition, OCT1/2 deficiency was not associated with compensatory expression changes in phylogenetically linked transporters (Figure 2A), with the exception of OCT3. Although ОСТ3 is expressed more abundantly than OCT2 in DRGs (Figure 1A), OCT3 deficiency failed to offer sustained protection against OIPN 24 hours after treatment (Supplemental Figure 1D), suggesting that ОCT3 did

\section{Results and Discussion}

In order to gain preliminary insight into the relative contribution of individual uptake transporters to OIPN, we performed a comparative transcriptomic profile of drug transporters in isolated DRGs from untreated mice (Figure 1A). Preliminary studies indicated that the expression and OIPN profile were similar between sexes and across different strains (Supplemental Figure 1, A-C; supplemental material available online with this article; https:// doi.org/10.1172/JCI136796DS1). Although copper transporter 1 (CTR1) had the highest expression in DRGs (Figure 1A), CTR1 was not considered as a candidate transporter because CRISPRmediated knockout of CTR1 in cells has demonstrated a CTR1independent mechanism of oxaliplatin accumulation (14). Based not independently contribute to the observed phenotypes. Candidate transporters were next evaluated for their ability to transport oxaliplatin in HEK293 cells overexpressing mouse and human homologous proteins. These studies indicate that OCT2 was the most efficient oxaliplatin transporter (Figure 1, D and E, and Supplemental Table 1), and lack of transport by OCT1 suggests that phenotypic alterations observed in OCT $1 / 2^{--}$mice are exclusively due to the deficiency of ОСТ2.

Histological examination of DRG cross sections revealed the absence of morphological damage in both WT and OCT1/2-mice, with minimal degeneration of caudal and sciatic nerve fibers (Supplemental Figure 2, A and B). Taken together with the lack of changes observed in nerve conduction studies (Supplemental Fig- 


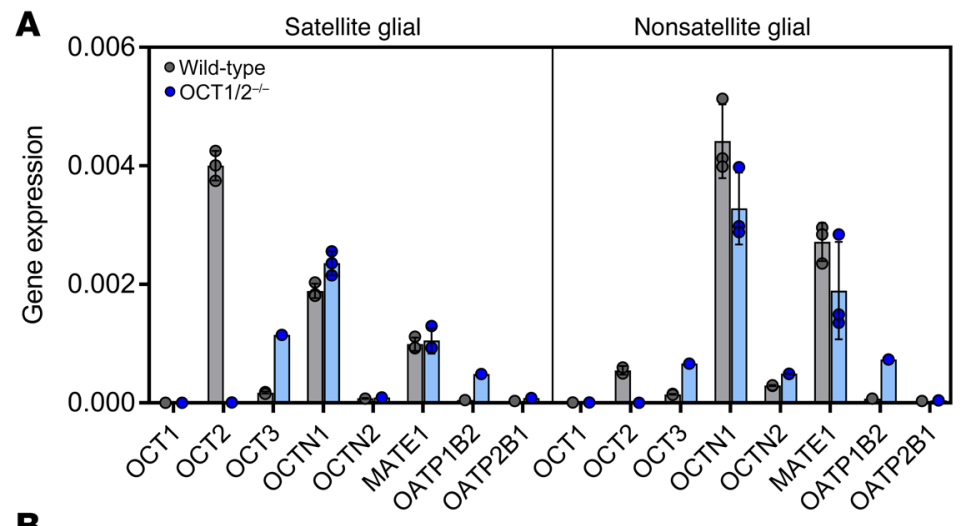

B
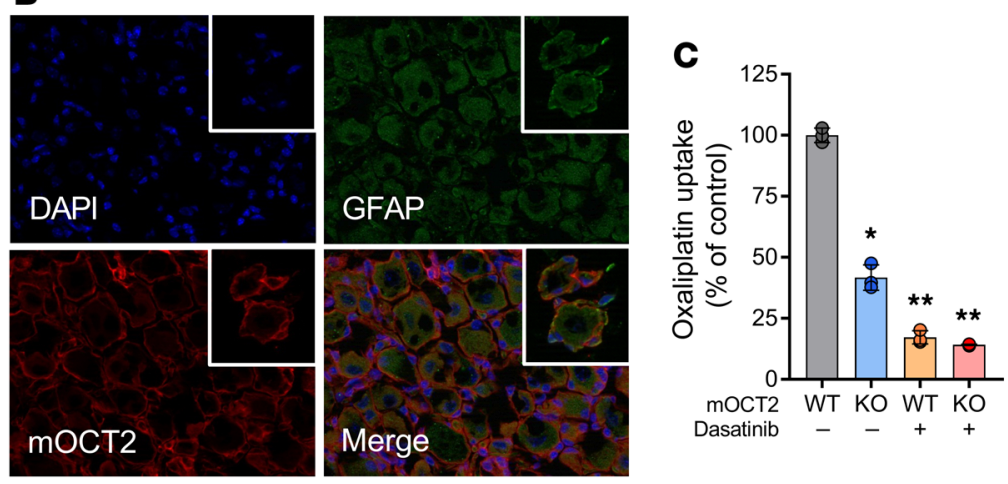

Figure 2. Localization and functional expression of OCT2 in satellite glial cells. (A) Expression of putative oxaliplatin carriers in primary satellite glial cells isolated from DRGs (left panel) compared with nonsatellite glial cell fractions (right panel) of untreated WT FVB mice. (B) Staining of DRG cross sections with DAPI (blue) and immunofluorescent staining for mOCT2 (red) or GFAP (green). Representative images at $\times 10$ and $\times 100$ original magnification. (C) Accumulation of oxaliplatin in primary SCCs isolated from untreated WT FVB or OCT1/2-1- mice with or without $10 \mu \mathrm{M}$ dasatinib pretreatment. Oxaliplatin uptake is expressed as percentage change compared with empty vector controls. Statistical analysis was performed using 1-way ANOVA with Dunnett's post hoc test: ${ }^{*} P<0.05,{ }^{* *} P<0.01$ compared with baseline values. ure $2 \mathrm{C}$ ), these findings suggest the development of mild ganglionopathy and support the notion that functional deficits in sensory transduction and neuronal firing of proprioceptors can continue to exacerbate behavioral outcomes despite the absence of tissue degeneration associated with OIPN (15). Because DRGs represent ganglia of sensory neuronal and glial cells, we next evaluated the contributing cell type to OIPN. Immunofluorescence staining of untreated DRG cross sections demonstrated the colocalization of OCT2 with glial fibrillary acidic protein (GFAP) (Figure 2B), a satellite glial cell (SGC) marker (16) that has been implicated in the development of chemotherapy-induced neuropathic pain (17). Subsequent investigation with primary cultures of SGCs indicated that OCT2 was highly expressed (Figure 2A) compared with nonsatellite cells (e.g., sensory neurons). Additionally, primary SGCs from OCT1 $/ 2^{-/-}$mice have diminished ability to accumulate oxaliplatin (Figure 2C and Supplemental Figure 3A). Similar findings were obtained in SGCs pretreated with dasatinib, a known inhibitor of OCT2 (Figure 2C and ref. 18).

Previously studies have indicated that targeting of OCT2 can confer protection against OIPN in mice $(10,18)$. In order to demonstrate the translational relevance of this concept (Supplemental Figure 3B), we next sought to validate the importance of OCT2 in OIPN in Sprague-Dawley rats as a secondary model organism (19). The utility of rats in OIPN was confirmed by the demonstration that rat OCT2 transports oxaliplatin and is highly sensitive to inhibition by dasatinib (Figure 3, A and B). The translational potential of using an OCT2 inhibitor as an intervention strategy to prevent the onset of OIPN was next evaluated in an acute model using WT and OCT2-deficient rats (Supplemental Figure $4, \mathrm{~A}-\mathrm{C}$ ) pretreated with orally administered dasatinib. These findings indicate that dasatinib significantly protected rats from OIPN (Figure 3C), similarly to that observed in OCT2deficient rats, and that OCT2 deficiency or dasatinib pretreatment did not significantly influence urinary excretion or plasma levels of oxaliplatin (Figure 3, D and E) but diminished accumulation of oxaliplatin in DRGs (Figure 3F). Similarly, dasatinib reduced the accumulation of oxaliplatin in primary WT rat SGCs (Figure 3G and Supplemental Figure 5, A-C), consistent with a previous murine report (18) and the current study.

In contrast with our current findings, it was previously suggested that the transporter OCTN1 is a possible determinant of OIPN $(6,12,20)$. Because of the expression of OCTN1 on the mitochondria, the neuroprotective effects of ergothioneine, an OCTN1-specific substrate, may be in part due to the contribution of intrinsic antioxidant activity to mitochondrial dysfunction rather than inhibition of oxaliplatin transport into DRGs. Our in vitro uptake studies indicate that oxaliplatin is not a transported substrate of mouse or human OCTN1 (Figure 1, D and E) and deficiency of OCTN1 or pretreatment with ergothioneine in mice did not afford neuroprotection (Figure 1B and Supplemental Figure 6A). A similar lack of oxaliplatin transport was reported for models overexpressing rat OCTN1 (21). It is conceivable that the neuroprotective effects associated with physiological concentrations of ergothioneine in chronic oxaliplatin treatment regimens are also partially due to effects on transporters other than OCTN1. Support for this comes from a prior study demonstrating that ergothioneine can significantly reduce the cellular uptake of oxaliplatin by more than $50 \%$ in DRG neurons from OCTN1-deficient mice (22). This earlier work also indicated that $80 \%$ of oxaliplatin uptake into DRG neurons occurs independently of OCTN1 and that oxaliplatin-induced loss of neuronal cell viability was unchanged in OCTN1-deficient mice 

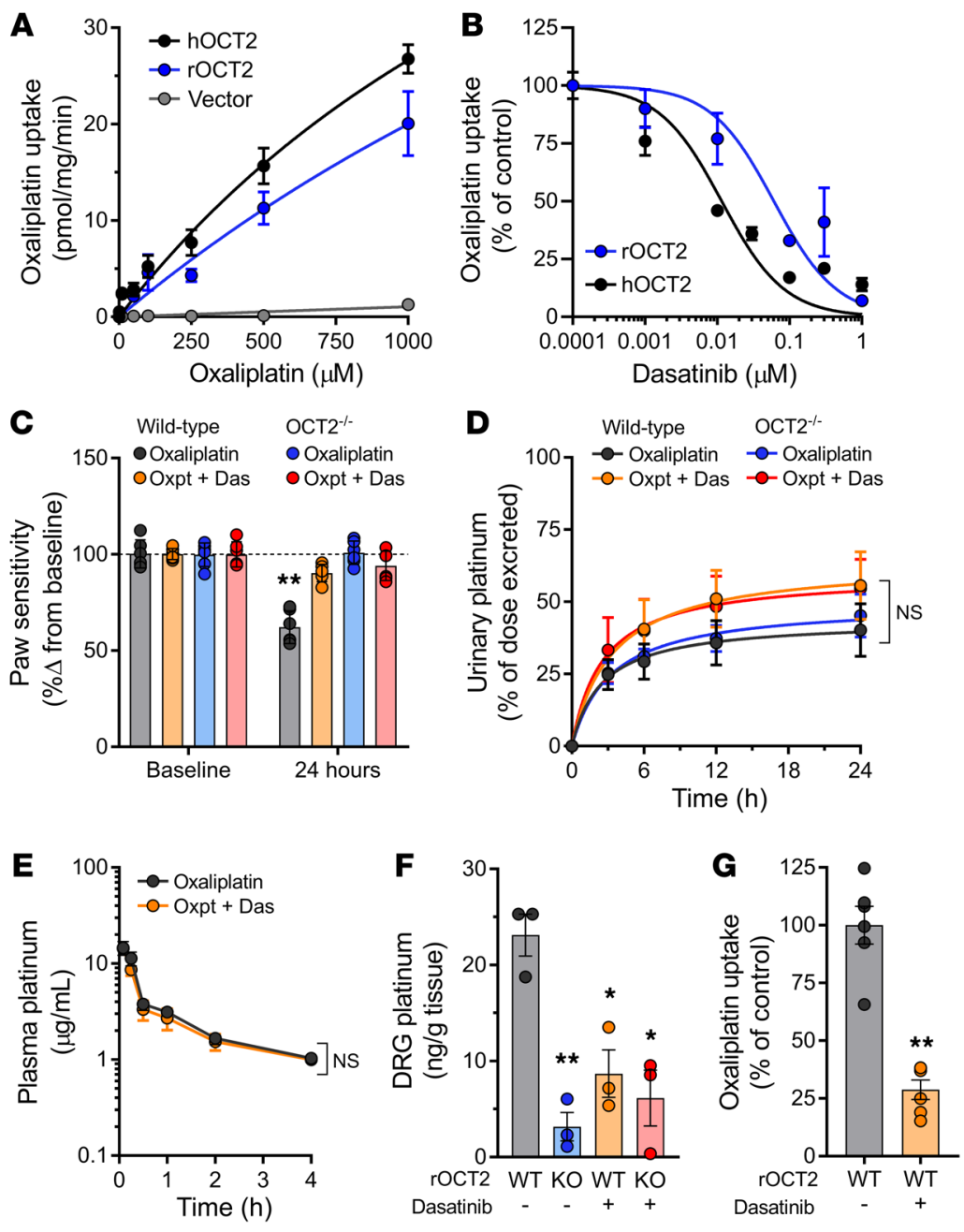

Figure 3. Genetic and pharmacologic targeting of OCT2 protects rats from OIPN. (A) Comparative transport kinetics of oxaliplatin in HEK293 cells overexpressing a rat $(r)$ or human (h) homolog of OCT2 ( $\mathrm{V}_{\text {max }}, 83.3$ and $94.4 \mathrm{pmol} / \mathrm{mg} / \mathrm{min}$ and $\mathrm{K}_{\mathrm{m}}, 2130$ and $3726 \mu \mathrm{M}$, respectively). (B) Sensitivity of rat and human $\mathrm{OCT} 2$ to inhibition by dasatinib, $\mathrm{IC}_{50} 60.4$ and 11.5 $\mathrm{nM}$, respectively. (C) OIPN in WT or OCT2-/- rats, expressed as percentage change relative to baseline values. (D) Urinary excretion, expressed as percentage of total administered platinum, and (E) systemic clearance of oxaliplatin in rats pretreated with vehicle or dasatinib. (F) Platinum concentrations from isolated rat DRGs. All studies represented in $\mathbf{C}-\mathbf{F}$ reflect response/exposure following a single injection of 10 $\mathrm{mg} / \mathrm{kg}$ oxaliplatin and/or pretreatment with oral $15 \mathrm{mg} / \mathrm{kg}$ dasatinib. (C) Accumulation of oxaliplatin in SGCs isolated from untreated WT rats. Statistical analysis was performed using 1-way ANOVA with Dunnett's post hoc test: ${ }^{*} P<0.05$ ${ }^{*} P<0.01$ compared with baseline values.
(22), in line with our toxicity evaluations using the same mouse model (Figure 1B and Supplemental Figure 6A). Interestingly, some studies have also suggested that oxaliplatin is a transported substrate of the carnitine transporter OCTN2; however, the significance of this observation is unclear because administration of an OCTN2 substrate, L-carnitine, does not afford tissue protection against oxaliplatin $(12,20)$. Furthermore, we found that dasatinib does not inhibit these transport mechanisms (Supplemental Figure 6, B and C).

Although the addition of dasatinib to oxaliplatin-based regimens could reduce the incidence and severity of OIPN, it is important to demonstrate that such intervention does not compromise the anticancer efficacy of oxaliplatin. Transcriptional profiling of drug transporters using RNA-seq data (23) revealed that OCT2 is expressed at very low levels in human colorectal tumors and colorectal cancer cell lines (Supplemental Figure 7, A-C), compared with human DRGs. Consistent with this observation, we found that cytotoxicity and cellular uptake (Figure 4A and Supplemental Figure 7D) of oxaliplatin in multiple colorectal cancer cell lines was not altered by dasatinib. These findings suggest that oxaliplatin is taken up into cancer cells independently of OCT2 and that this unknown mechanism is insensitive to dasatinib-mediated inhibition. The translational potential of this strategy was further verified by the observation that dasatinib did not influence the plasma levels or anticancer efficacy of oxaliplatin in mice bearing HCT116 tumor cells, while simultaneously preventing OIPN (Figure 4, B-G, and Supplemental Figure 7, E and F).

Because dasatinib is known to inhibit transporters of importance to the distribution of oxaliplatin into hepatocytes and renal tubular cells $(24,25)$, including OATP1B2 and MATE1, we confirmed that deficiency of OATP1B2 did not affect the clearance of oxaliplatin (Supplemental Figure 8, A-D). Consistent with a role of MATE1 as a major elimination mechanism of cationic compounds, it is conceivable that the inhibition of MATE1 efflux may lead to increased renal tubular accumulation of oxaliplatin and increased nephrotoxicity. Although MATE1 deficiency in mice was associated with diminished urinary excretion of oxaliplatin, pretreatment with dasatinib did not influence markers of renal dysfunction such as serum creatinine and blood urea nitrogen (Supplemental Figure 9, A-C). Furthermore, even high doses of oxaliplatin did not cause renal injury in MATE1-deficient mice, as evaluated by histological and biomarker examination (Supplemental Figure 9, D-F).

The combination of SRC kinase inhibitors such as dasatinib with oxaliplatin-containing regimens has been previously evaluated in patients with colorectal cancer (26). Although OIPN was still observed in patients receiving this combination, it is important to point out that the applied dasatinib dosing schedule was not opti- 
A

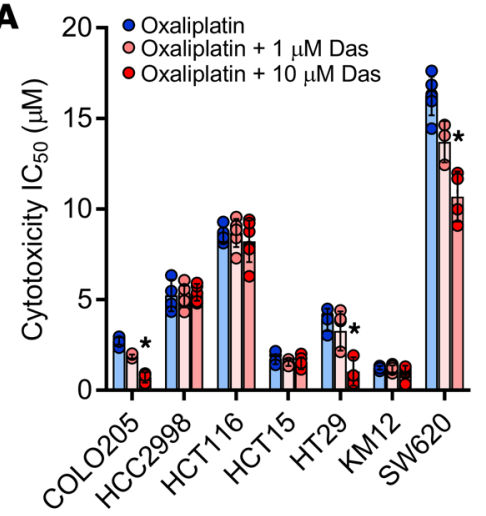

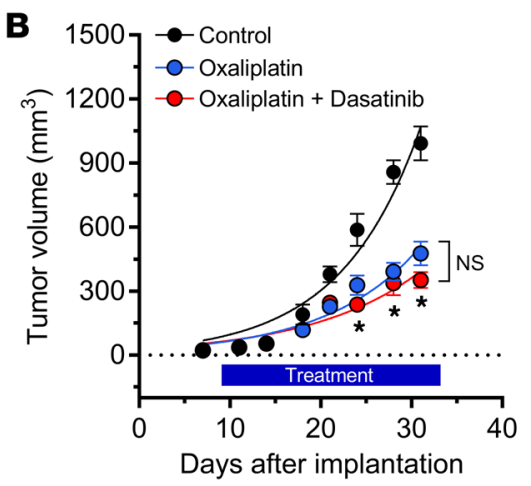

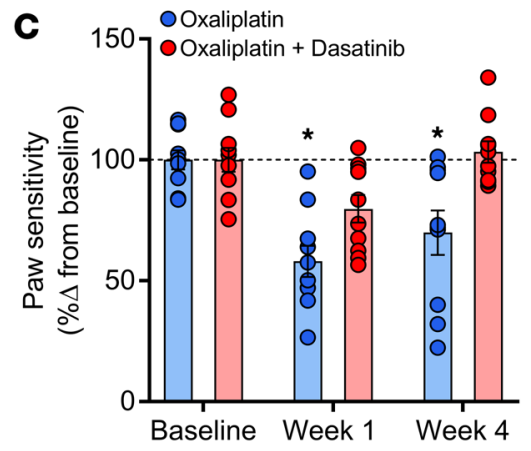

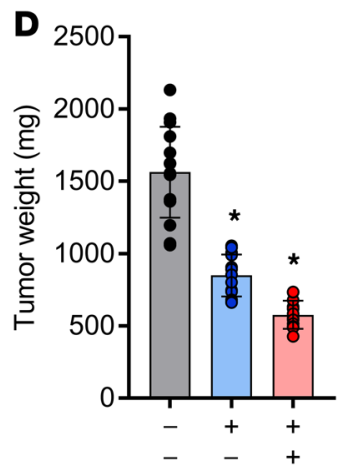
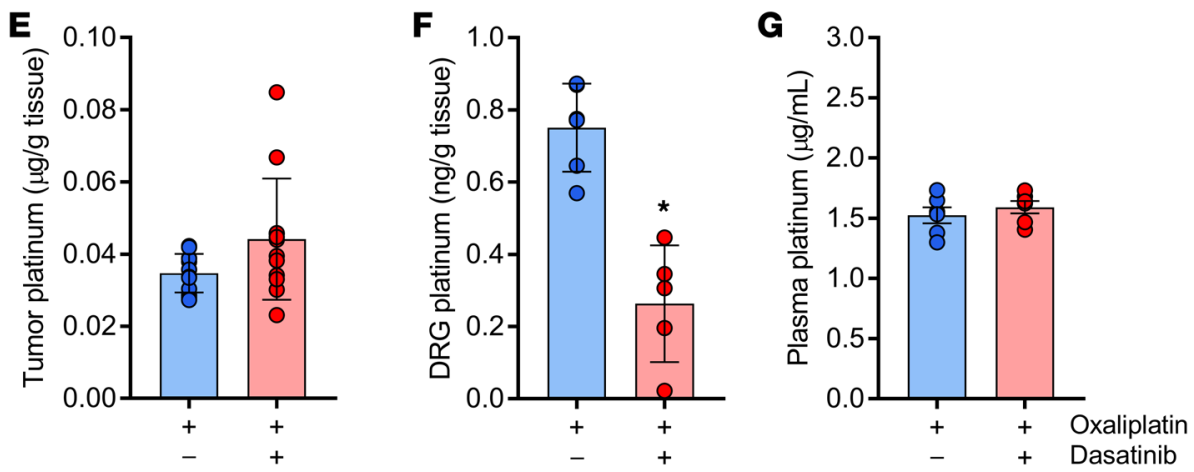

Figure 4. Dasatinib does not antagonize antitumor properties of oxaliplatin. (A) Cytotoxicity of oxaliplatin in the presence or absence of dasatinib (1 or 10 $\mu \mathrm{M}$ ) following 72-hour continuous exposure. (B) Tumor volume of immunocompromised nude mice subcutaneously inoculated with luciferase-expressing HCT116 cells. (C) OIPN in tumor-bearing mice at baseline, 1 week, or 4 weeks of treatment. (D) Quantification of tumor weight, and (E) platinum concentrations in tumors, (F) DRGs, or (G) plasma (30 minutes after final injection, representing steady-state concentrations). 0xaliplatin (4 mg/kg) was injected twice daily for 4 weeks (cumulative dose $32 \mathrm{mg} / \mathrm{kg}$ ); mice were pretreated with oral $15 \mathrm{mg} / \mathrm{kg}$ dasatinib 30 minutes before oxaliplatin. Paw sensitivity is expressed as percentage change from baseline values. Statistical analysis was performed using an unpaired, 2-sided Student's $t$ test with Welch's correction: ${ }^{*} P<0.05$ compared with baseline values.

mized in prior studies to exploit its OCT2-inhibitory properties. Our currently ongoing preclinical and clinical studies are designed to document local (DRG) and systemic (blood) changes in endogenous substrates of OCT2 that could be used as pharmacodynamic biomarkers of OCT2 function with inhibitors such as dasatinib.

In the context of our proposed intervention strategy, we acknowledge that several pharmacological approaches have been evaluated previously to prevent OIPN (27). Although duloxetine appears to be a viable lead from this collective work, the utility of this agent in preventing OIPN remains unclear (28). In a previously reported small-molecule library screen involving more than 8000 compounds (18), we found that more than half of the 433 compounds with potent OCT2 inhibitory properties were neurological compounds (Supplemental Figure 10, A-C). This is not surprising, considering the structural similarities of these compounds with known endogenous substrates of OCT2 such as catecholamines and neurotransmitters. Coincidentally, many of the OCT2 inhibitors identified from this screen, including duloxetine (Supplemental Figure 10D), have previously been evaluated in the management of neuropathic pain (27), based on their potential to inhibit intracellular signaling cascades that promote neuronal degeneration.

Collectively, we identified a previously unrecognized, SGCspecific pathway of oxaliplatin-induced neurological injury that is mediated by the transporter ОСТ2, which can be inhibited by dasatinib without compromising the anticancer properties of oxaliplatin. These findings not only shed light on the etiology of OIPN, but provide a rationale for future development of new targeted interventions using transport inhibitors to mitigate this debilitating side effect.

\section{Methods}

See Supplemental Methods for detailed methods.

Animal studies. All animals were housed in a temperature-controlled environment with a 12-hour light cycle, given standard chow diet and water ad libitum, and handled according to the Animal Care and Use Committee of The Ohio State University, under an approved protocol (2015A00000101-R1). For all experiments, age- and gendermatched WT or knockout mice (8-12 weeks) or rats (6-8 weeks) were used. Detailed information regarding sources and origins of the rodent models is provided in the Supplemental Methods.

Statistics. Data presented represent the mean \pm SEM before or after normalization to baseline values. All experiments were performed with replicates, unless stated otherwise, and repeated on at least 2 occasions. An unpaired, 2-sided Student's $t$ test with Welch's correction (2 groups) or a 1-way ANOVA with Dunnett's post hoc test ( $>2$ groups) was used to evaluate statistical significance using $P$ less than 0.05 as the cutoff. 


\section{Author contributions}

$\mathrm{SH}$ and $\mathrm{AS}$ conceived and designed the experiments. $\mathrm{KMH}, \mathrm{AFL}$, MEU, MC, AAG, KWH, SHX, and DD conducted in vitro experiments. KMH, AFL, JYK, YL, and SH carried out the in vivo experiments. EDE, YL, PA, AC, SNH, TCC, JAS, JW, CLL, AN, MBL, GC, and NP suggested experiments and contributed to data interpretation. AAG generated the stable cell lines. KMH, AFL, SH, and AS analyzed the data. KMH and AS wrote the manuscript. All authors have read and approved the final manuscript.

\section{Acknowledgments}

We would like to acknowledge the Small Animal Imaging Core at The Ohio State University for providing imaging instrumentation, and tissues procured by the National Disease Research Interchange (NDRI) supported by NIH grant 5U42RR006042. RNA sequencing data shown are based on publicly available data generated by TCGA Research Network (http://cancergenome.nih.gov). The project was supported in part by NIH grants R01CA221363 (to SNH and TCC), R01CA215802 (to AS), R01CA187176 (to AS), and R01CA238946 (to MBL and SH), and by the OSU Comprehensive Cancer Center using Pelotonia funds (to $\mathrm{KMH}$ ). The content is solely the responsibility of the authors and does not represent the official views of the funding agencies.

Address correspondence to: Shuiying Hu or Alex Sparreboom, Division of Pharmaceutics and Pharmacology, College of Pharmacy, The Ohio State University, BRT, Room 0406 (SH) or 0408 (AS), 500 West 12th Avenue, Columbus, Ohio 43210, USA. Phone: 614.685.8028; Email: hu.1333@osu.edu (SH). Phone: 614.685.6014; Email: Sparreboom.1@osu.edu (AS).
1. Argyriou AA, Polychronopoulos P, Iconomou G, Chroni E, Kalofonos HP. A review on oxaliplatininduced peripheral nerve damage. Cancer Treat Rev. 2008;34(4):368-377.

2. Pachman DR, et al. Clinical course of oxaliplatininduced neuropathy: results from the randomized phase III trial N08CB (Alliance). J Clin Oncol. 2015;33(30):3416-3422.

3. Thompson SW, Davis LE, Kornfeld M, Hilgers RD, Standefer JC. Cisplatin neuropathy. Clinical, electrophysiologic, morphologic, and toxicologic studies. Cancer. 1984;54(7):1269-1275.

4. Gregg RW, et al. Cisplatin neurotoxicity: the relationship between dosage, time, and platinum concentration in neurologic tissues, and morphologic evidence of toxicity. JClin Oncol. 1992;10(5):795-803.

5. Sprowl JA, Ness RA, Sparreboom A. Polymorphic transporters and platinum pharmacodynamics. Drug Metab Pharmacokinet. 2013;28(1):19-27.

6. Jong NN, Nakanishi T, Liu JJ, Tamai I, McKeage MJ. Oxaliplatin transport mediated by organic cation/carnitine transporters OCTN1 and OCTN2 in overexpressing human embryonic kidney 293 cells and rat dorsal root ganglion neurons. J Pharmacol Exp Ther. 2011;338(2):537-547.

7. Zhang $S$, et al. Organic cation transporters are determinants of oxaliplatin cytotoxicity. Cancer Res. 2006;66(17):8847-8857.

8. Filipski KK, Mathijssen RH, Mikkelsen TS, Schinkel AH, Sparreboom A. Contribution of organic cation transporter 2 (OCT2) to cisplatin-induced nephrotoxicity. Clin Pharmacol Ther. 2009;86(4):396-402.

9. Sprowl JA, et al. Oxaliplatin-induced neurotoxicity is dependent on the organic cation transporter OCT2. Proc Natl Acad Sci U S A. 2013;110(27):11199-11204.

10. Sprowl JA, et al. Conjunctive therapy of cisplatin with the OCT2 inhibitor cimetidine: influence on antitumor efficacy and systemic clearance. Clin Pharmacol Ther. 2013;94(5):585-592.

11. Li S, Chen Y, Zhang S, More SS, Huang X, Giacomini KM. Role of organic cation transporter 1, OCT1 in the pharmacokinetics and toxicity of cis-diammine(pyridine)chloroplatinum(II) and oxaliplatin in mice. Pharm Res. 2011;28(3):610-625.

12. Nishida K, et al. Ergothioneine ameliorates oxaliplatin-induced peripheral neuropathy in rats. Life Sci. 2018;207:516-524.

13. Lancaster CS, Sprowl JA, Walker AL, Hu S, Gibson AA, Sparreboom A. Modulation of OATP1B-type transporter function alters cellular uptake and disposition of platinum chemotherapeutics. Mol Cancer Ther. 2013;12(8):1537-1544.

14. Liu JJ, et al. Contributions of rat Ctr1 to the uptake and toxicity of copper and platinum anticancer drugs in dorsal root ganglion neurons. Biochem Pharmacol. 2013;85(2):207-215.

15. Vincent JA, Wieczerzak KB, Gabriel HM, Nardelli P, Rich MM, Cope TC. A novel path to chronic proprioceptive disability with oxaliplatin: distortion of sensory encoding. Neurobiol Dis. 2016;95:54-65.

16. Nascimento DSM, Castro-Lopes JM, Neto FLM. Satellite glial cells surrounding primary afferent neurons are activated and proliferate during monoarthritis in rats: is there a role for ATF3? PLoS One. 2014;9(9):e108152.

17. Warwick RA, Hanani M. The contribution of satellite glial cells to chemotherapy-induced neuropathic pain. Eur J Pain. 2013;17(4):571-580.

18. Sprowl JA, et al. A phosphotyrosine switch regulates organic cation transporters. Nat Commun. 2016;7:10880.

19. Ellenbroek B, Youn J. Rodent models in neuroscience research: is it a rat race? Dis Model Mech. 2016;9(10):1079-1087.
20. Fujita S, Hirota T, Sakiyama R, Baba M, Ieiri I. Identification of drug transporters contributing to oxaliplatin-induced peripheral neuropathy. J Neurochem. 2019;148(3):373-385.

21. Tschirka J, Kreisor M, Betz J, Gründemann D. Substrate selectivity check of the ergothioneine transporter. Drug Metab Dispos. 2018;46(6):779-785.

22. Jong N. The role of organic cation/carnitine transporters in the uptake and neurotoxicity of oxaliplatin. Doctoral thesis. The University of Auckland; 2012. http://hdl.handle.net/2292/19750.

23. Cancer Genome Atlas Research Network, et al. The Cancer Genome Atlas Pan-Cancer analysis project. Nat Genet. 2013;45(10): 1113-1120.

24. Minematsu T, Giacomini KM. Interactions of tyrosine kinase inhibitors with organic cation transporters and multidrug and toxic compound extrusion proteins. Mol Cancer Ther. 2011;10(3):531-539.

25. Zimmerman EI, et al. Contribution of OATP1B1 and OATP1B3 to the disposition of sorafenib and sorafenib-glucuronide. Clin Cancer Res. 2013;19(6):1458-1466.

26. Strickler JH, et al. Phase I study of dasatinib in combination with capecitabine, oxaliplatin and bevacizumab followed by an expanded cohort in previously untreated metastatic colorectal cancer. Invest New Drugs. 2014;32(2):330-339.

27. Hu S, Huang KM, Adams EJ, Loprinzi CL, Lustberg MB. Recent developments of novel pharmacologic therapeutics for prevention of chemotherapy-induced peripheral neuropathy. Clin Cancer Res. 2019;25(21):6295-6301.

28. Smith EM, et al. Effect of duloxetine on pain, function, and quality of life among patients with chemotherapy-induced painful peripheral neuropathy: a randomized clinical trial. JAMA. 2013;309(13):1359-1367. 